\title{
Effect of Oral Administration of Dietary Antioxidant Supplements in Patients with Chronic Hepatitis C
}

\author{
Torricelli Piera', Antonelli Francesco², Ferorelli Pasquale ${ }^{2}$, De Martino Angelo², Shevchenko Anna ${ }^{3}$, \\ Beninati Simone $^{2}$
}

${ }^{1}$ Department SPES, University of Molise, Campobasso, Italy

${ }^{2}$ University of Tor Vergata, Department of Biology, Rome, Italy

${ }^{3}$ People's Friendship University of Russia, Department of Sciences, Moscow, Russia

\author{
Email address: \\ beninati@bio.uniroma2.it (S. Beninati)
}

\section{To cite this article:}

Torricelli Piera, Antonelli Francesco, Ferorelli Pasquale, De Martino Angelo, Shevchenko Anna, Beninati Simone. Effect of Oral Administration of Dietary Antioxidant Supplements in Patients with Chronic Hepatitis C. American Journal of Clinical and Experimental Medicine. Vol. 3, No. 4, 2015, pp. 137-141. doi: 10.11648/j.ajcem.20150304.12

\begin{abstract}
In a prospective, randomized and single-blinded clinical trial, we compared patients with Chronic hepatitis C (CHC) orally treated with sucrose diluted with water (1:2) twice a day for 56 days (control group), with patients orally treated with four antioxidant dietary supplements rich in vitamin B5, B9, C, D, citric, pyruvic, and tartaric acids and carbohydrates (CCEP: Citexivir, Citozym, Ergozym Plus and Propulzym). The efficacy of treatment was evaluated once a week for 8 weeks, by monitoring changes in the activities of circulating Alanine aminotransferase (ALT), Aspartate aminotransferase (AST) and Gamma-glutamyl transferase (GGT) as markers of liver damage. After a treatment of 84 days the viral title was evaluated through the HCV-RNA compared with the levels of anti-C100-3. The security and tolerability of the treatment were evaluated on the basis of clinical adverse events and results of laboratory tests. The experimental data obtained showed that the oral treatment of patients suffering from HCV infection of genotype 1, with CCEP, markedly influenced the values of the three enzymatic markers of hepatic disease. The data presented also showed the reduction of viral replication evidenced by the rate of HCV-RNA levels. As reported by others, we confirmed the low reliability of the research of anti-C100-3. This research is not meant to suggest the treatment reported as a therapy for the treatment of HCV infection, but data obtained may tend towards the possibility of administration of a dietary supplement such as CCEP in support of the official drug therapy of CHC in the nutritional care of $\mathrm{HCV}$ patients.
\end{abstract}

Keywords: Antioxidant Food Supplements, Oxidative Stress, Chronic Hepatitis C, HCV

\section{Introduction}

Hepatitis $\mathrm{C}$ virus (HCV) infection is considered an important public health problem [1] and is the leading cause of liver transplantation in the Western world. Chronic HCV infection increases the risk for hepatic steatosis, insulin resistance, glucose intolerance and type 2 diabetes[2].

The molecular mechanism of HCV pathogenesis and the cause of progression of liver disease to severe liver injury are still poorly understood. Oxidative stress has emerged as a key player in the development and pathogenesis of chronic HCV [3]. Indeed, several experimental evidences suggest that oxidative stress contributes to steatohepatitis and that the increased generation of reactive oxygen and nitrogen species, together with the decreased antioxidant defense, promotes the development of hepatic and extrahepatic complications of $\mathrm{HCV}$ infection. The principal mechanism causing oxidative stress in HCV-positive subjects is derived by the direct activity of $\mathrm{HCV}$ proteins in increasing mitochondrial ROS production, as key events [4]. Cells are protected against oxidative insults by natural antioxidant products, notably glutathione, and by diverse antioxidant enzymes such as superoxide dismutase (SOD), catalase (CAT) and glutathione peroxidase (GSHPx) [5]. Oxidative stress develops when the disturbance in balance between the reactive oxygen forms produced in excess, and the factors preventing their harmful effect occurs.

Antioxidants and other strategies that decrease reactive oxygen (ROS) and nitrogen species (RNS), have been evaluated in the treatment of CHC. Vitamin C, E, D, silybin- 
phospholipids/vitamin E complex, and mitoquinone could each help alleviate the plasma or serum ALT, albeit to varying degrees in patients, at least before the cessation of therapy [6-8]. $\alpha$-Tocopherol could also prevent hepatic stellate cell activation in hepatitis $\mathrm{C}$ patients [9]. Certain antioxidant cocktails used in the absence of concurrent Interferon $\alpha$ (IFN) therapy likewise produced favorable effects on ALT in patients [10].

Considering these factors, appropriate nutrition becomes an essential tool to minimize HCV's comorbidities such as fatty liver, inflammation and insulin resistance. Recently, functional foods have been considered essential for promoting and maintaining health [11]. The aim of this study was to evaluate the influence of some food supplements with an enhanced antioxidative capability on ALT, AST and GGT levels in non-diabetic $\mathrm{CHC}$.

Our objective was extended to the evaluation of the viral replication by detection of the "viral load" under this antioxidative treatment performed by four dietary supplements with reported antioxidant properties [12].

\section{Materials and Methods}

\subsection{Patients}

Non-diabetic patients with CHC, aged between 35 and 60 years were recruited from a reference ambulatory unit of the University. The diagnosis of HCV infection was made by the presence of serum anti-HCV, which was confirmed by qualitative determination of HCV-RNA. Inclusion criteria were the following: patients aged over 18 years, with or without liver cirrhosis; patients with ethanol consumption below $20 \mathrm{~g} / \mathrm{d}$; patients with normal liver function and patients who were under antiviral therapy with IFN and Ribavirin or Telaprevir. Patients co-infected with HIV and/or HBV with renal failure as well as those with heart disease, decompensated cirrhosis, pregnancy, any malignancy, diabetes mellitus or obesity were excluded. The subjects gave written informed consent before participating in the study. The Ethics Committee of the University approved the study.

\subsection{Experimental Protocol}

The study was a prospective, randomized and singleblinded clinical trial. Subjects who met the inclusion criteria of the study were randomly allocated into one of two study groups. The study was single-blinded and the estimated sample size was 36 patients.

The 36 patients were equally divided into two groups $(\mathrm{n}=$ 18 ), and each group was composed of males with chronic hepatitis histologically confirmed, by infection with $\mathrm{HCV}$ genotype 1 (frequency ca. 60\%), and persistently elevated ALT, AST and GGT. The control group (A) was supplemented with sucrose diluted in water at $50 \%(\mathrm{w} / \mathrm{v})$ and the intervention group (B) was supplemented with the four dietary antioxidant supplements according to the reported protocol. In the absence of contraindications to the four supplements, group B patients were administered in increasing doses during the planned 56 days of treatment. Therefore, for the first 5 days of treatment, $10 \mathrm{~mL}$ of Citexivir before breakfast and after dinner. For the next 10 days, $10 \mathrm{~mL}$ of Citexivir before breakfast and $6.5 \mathrm{~g}$ of Ergozym Plus during breakfast. At night $20 \mathrm{~mL}$ of Citexivir dissolved in water. From day 16th until day 23rd, 6.5 g. of Ergozym Plus during breakfast and $60 \mathrm{~mL}$ of Citexivir dissolved in $500 \mathrm{~mL}$ of water to drink during the day. Finally, from day 24 until the end of treatment (day 56th), $6.5 \mathrm{~g}$ of Ergozym Plus for breakfast and $60 \mathrm{~mL}$ of Citexivir dissolved in $500 \mathrm{~mL}$ of water to drink during the day. After dinner 25 $\mathrm{mL}$ of Citozym along with $6.5 \mathrm{~g}$ of Propulzym dissolved in water. An accurate observation of possible adverse effects on the patient was performed. Citexivir, Citozym, Ergozym Plus and Propulzym, were obtained from CITOZEATEC, S.r.1. (Peschiera Borromeo, Milano, Italy).

Patients were instructed to dissolve the supplement in water, juice, soup, porridge or to consume it with fruits. Additionally, considering their nutritional status and dietary habits, patients received dietary guidelines to promote healthy eating and weight control. Diet counseling, aimed to promote the ingestion of a normocaloric, normoglycidic and normoproteic diet by both groups was performed .

\subsection{Observation and Evaluation of Clinical Signs}

Clinical survey data such as clinical diagnosis, viral genotype, necroinflammatory activity index and fibrosis (METAVIR classification) were either collected from medical records or from patient examinations. Patients underwent ultrasonography of the upper abdomen with a team of three examiners using a single piece of equipment at the University Hospital's radiology service. Hepatic steatosis was graded as mild, moderate or severe according to the classification of Saverymuttu et al [13] .

Measurement of waist circumference was performed according to the World Health Organization recommendations using an inelastic tape measure (TBW Import Ltd.) that was $0.5 \mathrm{~cm}$ wide and $200 \mathrm{~cm}$ in length. Waist circumference was measured at a level midway between the superior aspect of the iliac crests and the lower lateral margins of the ribs. The cutoff points adopted for classifying central obesity and increased risk of metabolic complications were above $80 \mathrm{~cm}$ for women and $94 \mathrm{~cm}$ for men [14]. Socio-demographic and lifestyle information were also collected using a structured questionnaire during the first appointment of follow-up.

Patients underwent follow-up visits once a week, with registered dietitians to elucidate the adherence to the diet prescription and antioxidant dietary supplements protocol. Ultrasound was also performed in patients with a diagnosis of hepatic steatosis at baseline.

\subsection{Bioassays}

After a 12-h fast, a blood sample was collected weekly for the determination of AST, ALT and GGT. Analyses were performed on a Beckman Coulter LX-20 PRO and CX-9 
equipment. A qualitative and quantitative Hepatitis C RNA tests were used. The quantitative test performed by PCR, measures the number of copies of HCV RNA in the blood, also known as the "viral load." Quantitative measurement of "viral load" was carried out using the HCV Ampliprep TaqMan, Roche Molecular System, at the same time as ALT determination [15-16].

\subsection{Statistical Analysis}

Descriptive analysis was performed to characterize the population. Mann-Whitney U and Wilcoxon's rank sum tests were used to compare the biochemical values in the intervention and control groups and to evaluate the differences obtained at baseline and after intervention. Logistic regression analysis was used to evaluate risk predictor factors for hepatic steatosis, insulin resistance (HOMA-IR $\geq 3.0$ ) and changes in ALT levels (1.5 above the upper limit of normal). Confounding and interaction analyses were performed to select the final models. Multiple regression analysis was performed after the intervention considering the groups had similar demographic, clinical and laboratory features before CCEP supplementation.

The sample size was calculated using an estimated $20 \%$ loss to follow-up, a confidence level of $95 \%$ and $80 \%$ power. A statistical significance was inferred at $\mathrm{p}<0.05$. In some biochemical analyses, the Bonferroni method was applied to adjust the multiple comparisons p-value between the groups at the significance level of 0.007 . Statistical analysis was performed with the statistical package $\mathrm{R}$ version 2.12 .

\section{Results}

After 56 days of CCEP supplementation (Table I), a significant reduction was observed in the transaminase levels of the group B vs the group A (AST: $50 \%, \mathrm{p}<0.05$; ALT: $38 \%, \mathrm{p}<0.05)$. A remarkable reduction in the levels of GGT was also observed in the group B compared to the group A $(71 \%, \mathrm{p}<0.05)$.

Table I. values of the enzymatic activities obtained during 8 weeks of treatment with CCEP of patients with CHC. In parentheses the values of the control group not treated. Data represent the mean of 18 determinations with a S.D. lower than $15 \%$.

\begin{tabular}{lccl}
\hline WEEKS & AST (IU/L) & ALT (IU/L) & GGT (IU/L) \\
\hline 1 & $85 \pm 5(90)$ & $139 \pm 12(140)$ & $106 \pm 10(132)$ \\
2 & $60 \pm 7(95)$ & $140 \pm 15(140)$ & $92 \pm 6(115)$ \\
3 & $75 \pm 7(70)$ & $160 \pm 16(160)$ & $120 \pm 9(146)$ \\
4 & $65 \pm 6(75)$ & $125 \pm 13(150)$ & $95 \pm 8(157)$ \\
5 & $80 \pm 4(80)$ & $110 \pm 10(125)$ & $50 \pm 6(123)$ \\
6 & $45 \pm 2(60)$ & $90 \pm 5(130)$ & $33 \pm 2(137)$ \\
7 & $50 \pm 2(70)$ & $75 \pm 4(130)$ & $48 \pm 3(143)$ \\
8 & $35 \pm 2(70)$ & $74 \pm 7(120)$ & $36 \pm 4(123)$ \\
\hline
\end{tabular}

The values presented in Figure 1, were obtained from the average of the 18 values of activity of each group analyzed for each week. The variations from the average never exceeded $15 \%$. Group B showed the reduction of the enzyme values for ALT and GGT starting from the 4th week of treatment. AST activity starts to decrease from the 6th week of treatment. The enzyme values related to AST normally did not undergo large changes during $\mathrm{HCV}$ infection. Obviously, the data can vary in individuals. The values related to the enzymatic activity of GGT, normally underwent large variations during infection by $\mathrm{HCV}$ and the pharmacological treatment for $\mathrm{HCV}$ infection, failed to reduce such enzyme levels in a short time.
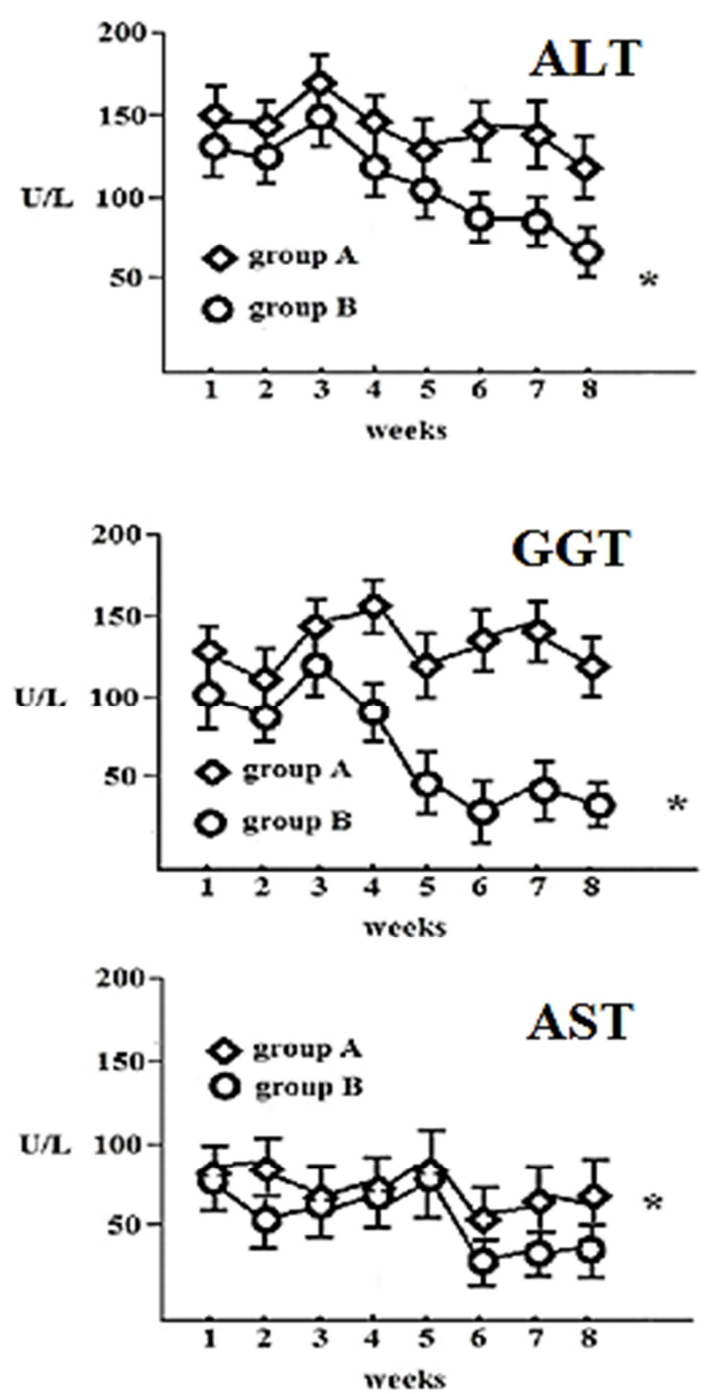

Figure 1. reduction of enzyme levels in patients with CHC treated with CCEP for 8 weeks Data represent the mean of 18 determinations with a S.D. lower than $15 \%$ * normal values.

After 84 days of CCEP supplementation, treated patients ( $\mathrm{n}=18$ units) and untreated ( $\mathrm{n}=18$ units) were assayed for the HCV-RNA, AST, ALT and GGT. The RNA of the Hepatitis C virus, comprises genes encoding for structural proteins and not. The HCV-RNA expression of virus replication, appears in serum few days before signs of liver damage, a few weeks or months before the onset of the anti-C 100-3. HCV-RNA is detected by means of the PCR technique. The test is extremely sensitive and therefore has high false positives. For its constant false positivity, the test must be repeated frequently to have a real evaluation of the course of disease. 
20 patients had initial values of HCV-RNA exceeding $1 \times 10^{6}$ I.U. / mL. 16 patients were in a range of HCV-RNA between $8 \times 10^{5}$ I.U. / mL and $1 \times 10^{6}$ I.U. / mL. Results in Table II are expressed as percentage of reduction compared to controls places to 100 . It shows a reduction of viral titer of $35 \%$ in group B when compared with group A. The percentage of reduction of the three activities examined appeared stabilized after 84 days of treatments at $46 \%$ for ALT, $80 \%$ for GGT and $59 \%$ for AST. A positive assay anti-C100-3 was obtained in $40 \%$ of cases observed and judged not significant in relation to the treatment carried out (data not shown).

Table II. Changes in percentage of enzyme values and the viral titer expressed as HCV-RNA in patients treated with CCEP for 84 days. The variability of the data did not exceed $15 \%$

\begin{tabular}{lll}
\hline data & group A & group B \\
\hline ALT & 100 & $46 \%$ \\
GGT & 100 & $80 \%$ \\
AST & 100 & $59 \%$ \\
HCV-RNA & 100 & $35 \%$ \\
\hline
\end{tabular}

\section{Discussion}

$\mathrm{HCV}$ infection is associated with severe alterations in the host redox status. [3]. Hepatic, blood, and lymphocytic glutathione is significantly depleted [17]. Decreases in plasma antioxidant potential and in the levels of vitamins A, $\mathrm{B}$, and $\mathrm{E}$, zinc, and selenium and significant increases in lipid peroxidation, oxidative DNA damage, and nitrotyrosine have also been found [18]. Several sources of reactive species that contribute to oxidative/nitrosative stress during HCV infection have been identified [3]. The oxidative DNA damage induced by HCV could be alleviated by strategies designed to prevent production of nitric oxide, suggesting potential roles of hydroxyl radical and peroxynitrite/nitration in the damage [19]. Liver is a net exporter of GSH. Therefore, it is conceivable that $\mathrm{HCV}$ will decrease the plasma and lymphocytic GSH indirectly through primary liver injury, decreasing the total GSH output [20]. In addition, HCV has been suggested to impair the host antioxidant defense specifically. Antioxidants and other strategies that decrease ROS/RNS have been evaluated in the treatment of $\mathrm{CHC}[8,9]$ Iron depletion, with or without IFN therapy, vitamin E, C and D could each help alleviate the plasma or serum ALT. Certain antioxidant cocktails used in the absence of concurrent IFN therapy likewise produced favorable effects on ALT in CHC patients [21].

The experimental data presented in our work, undertaken in order to restore the antioxidant properties of the HCV host, show that oral treatment with CCEP, a combination of four dietary antioxidant supplements [12, 22], of patients suffering from infection with HCV genotype 1, markedly influences the values of three enzymatic activities markers of hepatic disease (GGT,ALT and AST). This antioxidant treatment has also highlighted the reduction of viral replication assessed by the rate of HCV-RNA. Our research evidenced the low reliability of the values of anti-C100-3, as reported by several authors [23]. This research does not intend to suggest the treatment reported as a therapy for the treatment of $\mathrm{HCV}$ infection, but data obtained suggest the possibility of the use of CCEP as a dietary supplement to support the official therapy. In conclusion, CCEP supplementation decreased transaminases levels and thus may improve liver inflammation in $\mathrm{CHC}$ patients; it also reduced hepatic GGT in the treated group of patients but change marginally the virus title. The overall results of the experiment suggest that the blend of antioxidants used should be considered in the nutritional care of HCV patients.

\section{Acknowledgements}

We are grateful to A.I.A.S Italy, for financial support for studying the possibility of alternative therapies for $\mathrm{CHC}$.

\section{References}

[1] Lauer GM, Walker, B D Hepatitis C virus infection. N. Engl. J. Med. 2001; 345(1): 41-52.

[2] Pol S, Vallet-Pichard A, Corouge M, Mallet VO. Hepatitis C: epidemiology, diagnosis, natural history and therapy. Contrib Nephrol. 2012;176:1-9

[3] Seronello S, Sheikh M Y, Choi J. Redox regulation of hepatitis $\mathrm{C}$ in nonalcoholic and alcoholic liver. Free Radic. Biol. Med. 2007;43:869-882.

[4] Abdalla MY, Ahmad IM, Spitz DR, Schmidt WN, and Britigan BE. Hepatitis C virus-core and nonstructural proteins lead to different effects on cellular antioxidant defenses. J Med Virol 2005; 76: 489-497.

[5] Larrea E, Beloqui O, Munos-Navas MA, Civeira MP, Prieto J. Superoxidedismutase in patients with chronic hepatitis $\mathrm{C}$ virus infection. Free Radic Biol Med 1998; 24:1235-1241.

[6] von Herbay, A., et al. Vitamin E improves the aminotransferase status of patients suffering from viral hepatitis C: a randomized, double-blind, placebo-controlled study. Free. Radic. Res. 1997; 27:599-605.

[7] Mahmood, S., et al. Effect of vitamin E on serum aminotransferase and thioredoxin levels in patients with viral hepatitis C. Free. Radic. Res. 2003; 37: 781-785.

[8] Falasca, K., et al. Treatment with silybin-vitamin Ephospholipid complex in patients with hepatitis $\mathrm{C}$ infection. J. Med. Virol. 2008; 80:1900-1906.

[9] Houglum, K., et al. A pilot study of the effects of D- $\alpha$ tocopherol on hepatic stellate cell activation in chronic hepatitis C. Gastroenterology. 1997; 113:1069-1073.

[10] Melhem, A., et al. Treatment of chronic hepatitis $\mathrm{C}$ virus infection via antioxidants: results of a phase I clinical trial. J. Clin. Gastroenterol. 2005; 39:737-742.

[11] Morisco F, Vitaglione P, Carbone A, Stingo S, Scarpati S, Ascione A, Marmo R, Fogliano V, Caporaso N. Tomato-based functional food as interferon adjuvant in HCV eradication therapy. J Clin Gastroenterol. 2004;38(6):S118-120. 
[12] P. Torricelli, P. Ferorelli, A. De Martino, F. Antonelli, A. Shevchenko, S. Beninati. Regression of Carotid Plaques in Individuals at Low-to-intermediate Cardiovascular Risk Treated with Citozym and Propulzym. European Journal of Preventive Medicine. 2014; 2 (3): 33-37.

[13] Saverymuttu SH, Joseph AE, Maxwell JD. Ultrasound scanning in the detection of hepatic fibrosis and steatosis. $\mathrm{Br}$ Med J (Clin Res Ed).1986;292:13-15.

[14] Han TS, van Leer EM, Seidell JC, Lean ME. Waist circumference action levels in the identification of cardiovascular risk factors: prevalence study in a random sample. BMJ. 1995;311:1401-1405.

[15] Clancy A, Crowley B, Niesters H, Herra C. The development of a qualitative real-time RT-PCR assay or the detection of hepatitis $\mathrm{C}$ virus. Eur $\mathrm{J}$ Clin Microbiol Infect Dis. 2008;27(12):1177-1182

[16] Butcher A, Aslam S, Hemyari P, Cowen U, Heilek G. HCV RNA detection in HCV antibody-positive patients with the COBAS AmpliPrep/COBAS TaqMan HCV test, v2.0 in comparison with FDA-approved nucleic acid tests. J Clin Virol. 2014;60(4):336-340.

[17] Barbaro, G., et al. Serum ferritin and hepatic glutathione concentrations in chronic hepatitis $\mathrm{C}$ patients related to the hepatitis C virus genotype. J. Hepatol. 1999; 30:774-782.
[18] Kageyama, F., et al. Successful interferon therapy reverses enhanced hepatic iron accumulation and lipid peroxidation in chronic hepatitis C. Am. J. Gastroenterol. 2000; 95:1041-1050.

[19] Machida, K., et al. Hepatitis C virus infection activates the immunologic (type II) isoform of nitric oxide synthase and thereby enhances DNA damage and mutations of cellular genes. 2004; J. Virol. 2004; 78:8835-8843.

[20] Barbaro, G., et al. Hepatic glutathione deficiency in chronic hepatitis C: quantitative evaluation in patients who are HIV positive and HIV negative and correlations with plasmatic and lymphocytic concentrations and with the activity of the liver disease. Am. J. Gastroenterol. 1996; 91:2569-2573.

[21] Gabbay, E., et al. Antioxidant therapy for chronic hepatitis C after failure of interferon $\alpha$ results of phase II randomized, double-blind placebo controlled clinical trial. World J. Gastroenterol. 2007; 13:5317-5323.

[22] Torricelli P., Ferorelli P., De Martino A., Antonelli F., Beninati S.. The Influence of Preventive Multiple Micronutrients Supplementation on Liver Steatosis in High-cholesterol Fed C57BL6/N Mice. American Journal of Life Sciences. 2013;1(2):55-60

[23] Morii K1, Shimomura H, Nakagawa H, Hasui T, Tsuji T. AntiC100-3 antibody status, viral genomic sequences, and clinical features in chronic hepatitic patients with hepatitis $\mathrm{C}$ virus RNA in sera. Acta Med Okayama. 1992;46(4):285-293. 